\title{
Frequency Behavior of a Quartz Crystal Microbalance (Qcm) in Contact with Selected Solutions
}

\author{
${ }^{1}$ Z.A. Talib, ${ }^{1}$ Z. Baba, ${ }^{3}$ S. Kurosawa,${ }^{1}$ H.A.A. Sidek, ${ }^{2}$ A. Kassim ${ }^{b}$ and ${ }^{1}$ W.M.M. Yunus \\ ${ }^{1}$ Department of Physics, ${ }^{2}$ Department of Chemistry, Faculty of Science, Universiti Putra Malaysia \\ 43400 Serdang, Selangor, Malaysia \\ ${ }^{3}$ Institute for Environmental Management Technology \\ National Institute of Advanced Industrial Science and Technology (AIST), Tsukuba, Japan
}

\begin{abstract}
A device was constructed to monitor viscosity of solutions using fundamental frequency of $9 \mathrm{MHz}$ and $10 \mathrm{MHz}$ quartz crystal. Piezoelectric quartz crystals with gold electrodes were mounted by O-ring in between liquid flow cell. Only one side of the crystal was exposed to the solutions which were pumped through silicon tube by a peristaltic pump. The measured frequency shift was observed in order to investigate the interfacial behavior of some selected solution in contact with one surface of Quartz Crystal Microbalance (QCM). An analysis of the interaction between an AT-cut quartz crystal microbalance and various liquid system of analytical interest is presented. The analysis which includes piezoelectric effects and other influences; liquid properties, experimental conditions and the characteristic of the solution are reported. Oscillation in distilled water is taken as a reference. The frequency change caused by the density $\left(\rho, \mathrm{gcm}^{-3}\right)$ and viscosity $\left(\eta, \mathrm{gcm}^{-1} \mathrm{~s}^{-1}\right)$ were found to be proportional to the square root of the product, $(\rho \eta)$. The result suggested that analysis of small frequency shifts during EQCM studies needs to account for changes in $\rho$ and $\eta$ of the solution. Generally, all the liquid tested showed an increment of the frequency shift with increasing content of solutes. For each solution, the frequency was recorded as the concentration increases from distilled water to a very concentrated solution. The frequency measurements carried out for saccharide solution produces the maximum changes of frequency shift compared with other solutions.
\end{abstract}

Key words: Quartz crystal microbalance (QCM), liquid sensor, saccharide

\section{INTRODUCTION}

Quartz crystal microbalance (QCM) is a wellestablished method for the measurement of small changes in mass, based on the relationship between changes in mass of material attached to the crystal and the oscillation frequency of the crystal. It has also been found that they also play an important role in probing interfacial processes or acoustic properties of liquids. The operation of a QCM relies on the excitation into mechanical resonance induced by an electrical field across the quartz crystal with two metal electrodes on opposite sides of the quartz crystal plate.

The AT-cut of quartz has long been the most commonly used cut for quartz crystal oscillator applications. New advances in QCM methodology has enable the use of the QCM principle while the quartz crystal is immersed in liquid media ${ }^{[1,2]}$. Konash and Bastiaans ${ }^{[3]}$ reported that a crystal with one electrode in contact with an organic solvent also oscillates. They found that the frequency depends on the density and the arrangement may be used as a detector for liquid chromatography. Kanazawa and Gordon ${ }^{[4]}$ presented a quantitative description of the influence of the liquid properties on the oscillation frequency. They determined the behavior of the crystal/fluid system by examining the coupling of the elastic shear waves in the crystal to the viscous shear wave in the liquid. Kurosawa et al. ${ }^{[5]}$ examined the oscillating frequencies in various solutions by comparing the oscillating frequencies in distilled water. They observed that $\Delta f$ or $\Delta f_{W}$ in solutions is a linear function of $\sqrt{\rho \eta}$, except for salt and high polymer solutions. We have expanded the same experiment but with different flow cell configuration and used it on some solutions of analytical interest. The frequency behavior of the QCM immerse in those solutions is reported in this paper.

\section{MATERIALS AND METHODS}

Selected solutions with various concentrations were prepared using analytical-reagent grade chemical and distilled water. The specific gravity and viscosities were measured by pycnometer and Ubblohde viscometer. We used AT-cut quartz discs with gold electrodes and fundamental frequencies of $9 \mathrm{MHz}$ and $10 \mathrm{MHz}$ (ICM Co., Oklahoma City, US) in all experiment. A flow cell was constructed in which one face of the crystal is in contact with the liquid.

Corresponding Author: Z.A. Talib, Department of Physics, ${ }^{2}$ Department of Chemistry, Faculty of Science, Universiti Putra Malaysia, 43400 Serdang, Selangor, Malaysia 


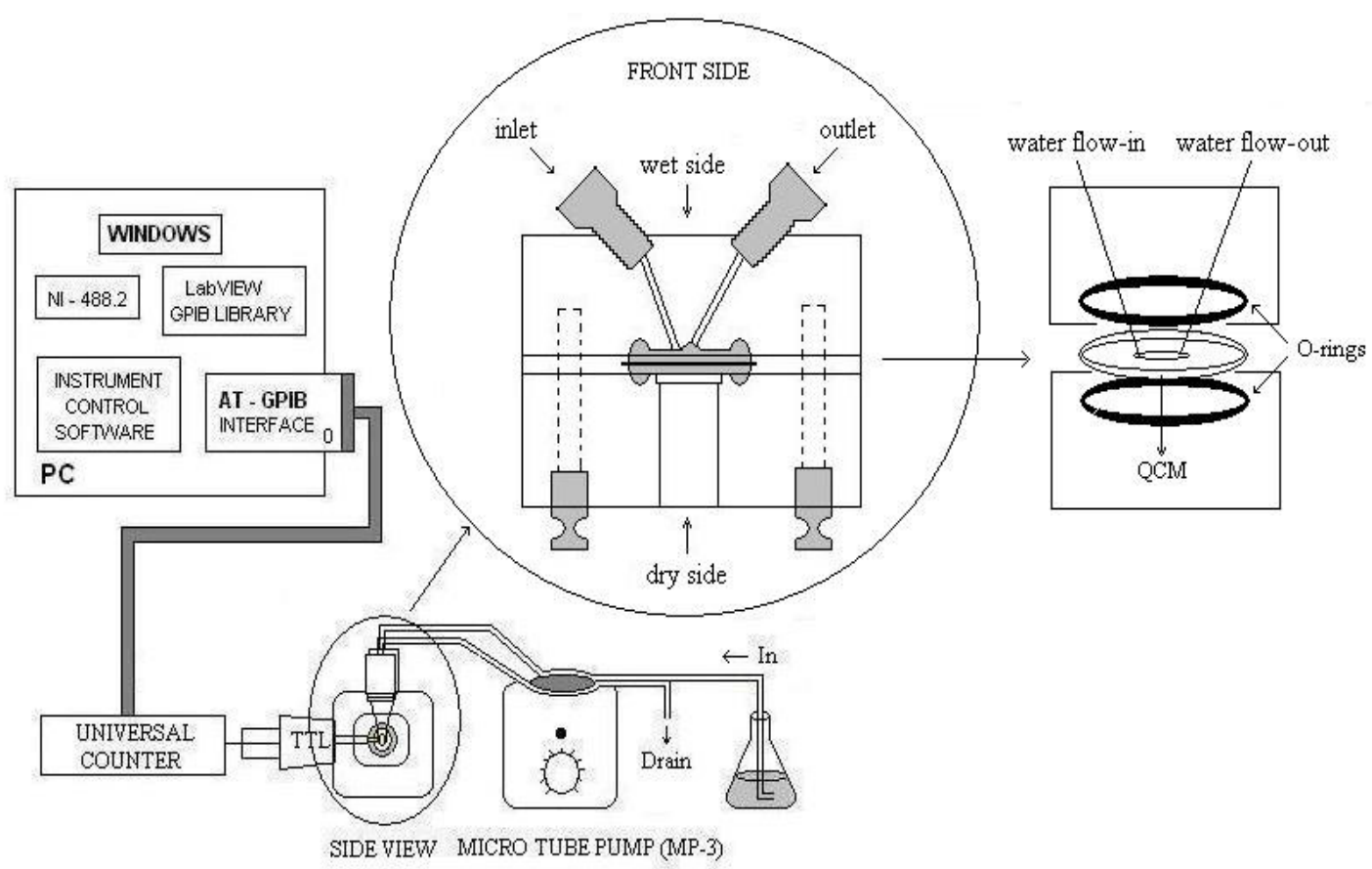

Fig. 1: Schematic diagram of the experimental setup. Test solutions were applied to the crystal by a micro-tubing pump through a silicon tube. Oscillating frequencies were measured with an Agilent Universal Counter (Model 5313A), which was connected to a microcomputer
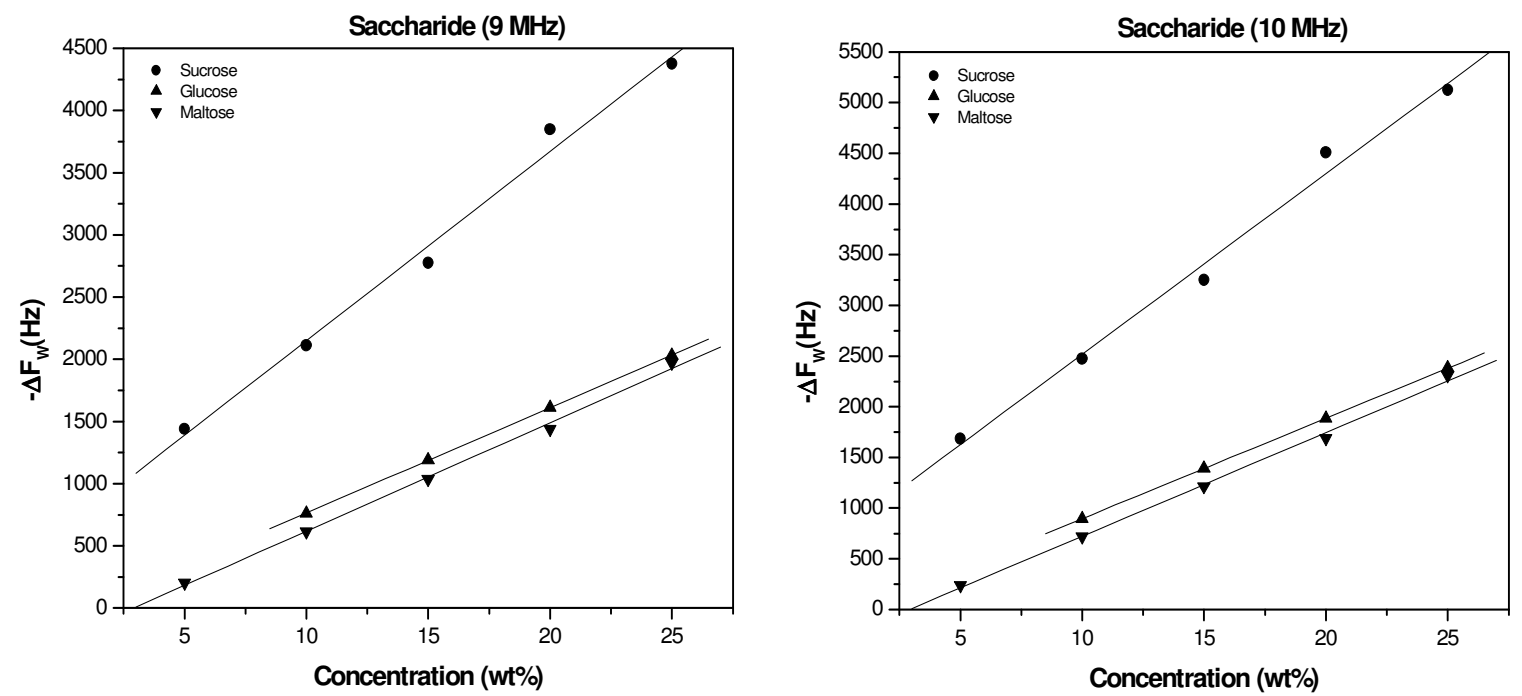

Fig. 2: Change in resonance frequency, relative to distilled water as a function of the concentration of saccharide in water

The crystals were clamped between two O-rings as shown in Fig. 1. Solutions are propelled to the flow cell by a micro-tubing pump, (MP-3, Eyela, Tokyo Rika) through $0.5 \mathrm{~mm}$ diameter silicon tube. The flow-rate was between 1.3-1.6 ml min ${ }^{-1}$ and no dependence of the flow-rate on the frequency change was found. The surface of the working electrode was cleaned in-situ by alcohol solution repeatedly after every experiment. The frequency change was monitored by a universal counter (Model 5313A, Agilent) which was monitored by a microcomputer with a GPIB interface programmed by LabVIEW.
After stabilization of the frequency with water, test solutions were applied stepwise from low to high concentrations. At the end of each experimental run, water was applied to check the reversibility of the frequency. The frequency of the crystal was measured every 5 seconds for all concentration of solutions. Owing to the low densities of pure ethanol and methanol, they were applied first, followed by test solutions mixed with water. Finally, water was applied. For water-immiscible organic solvent such as hexane, benzene and toluene, the oscillating frequency in water 

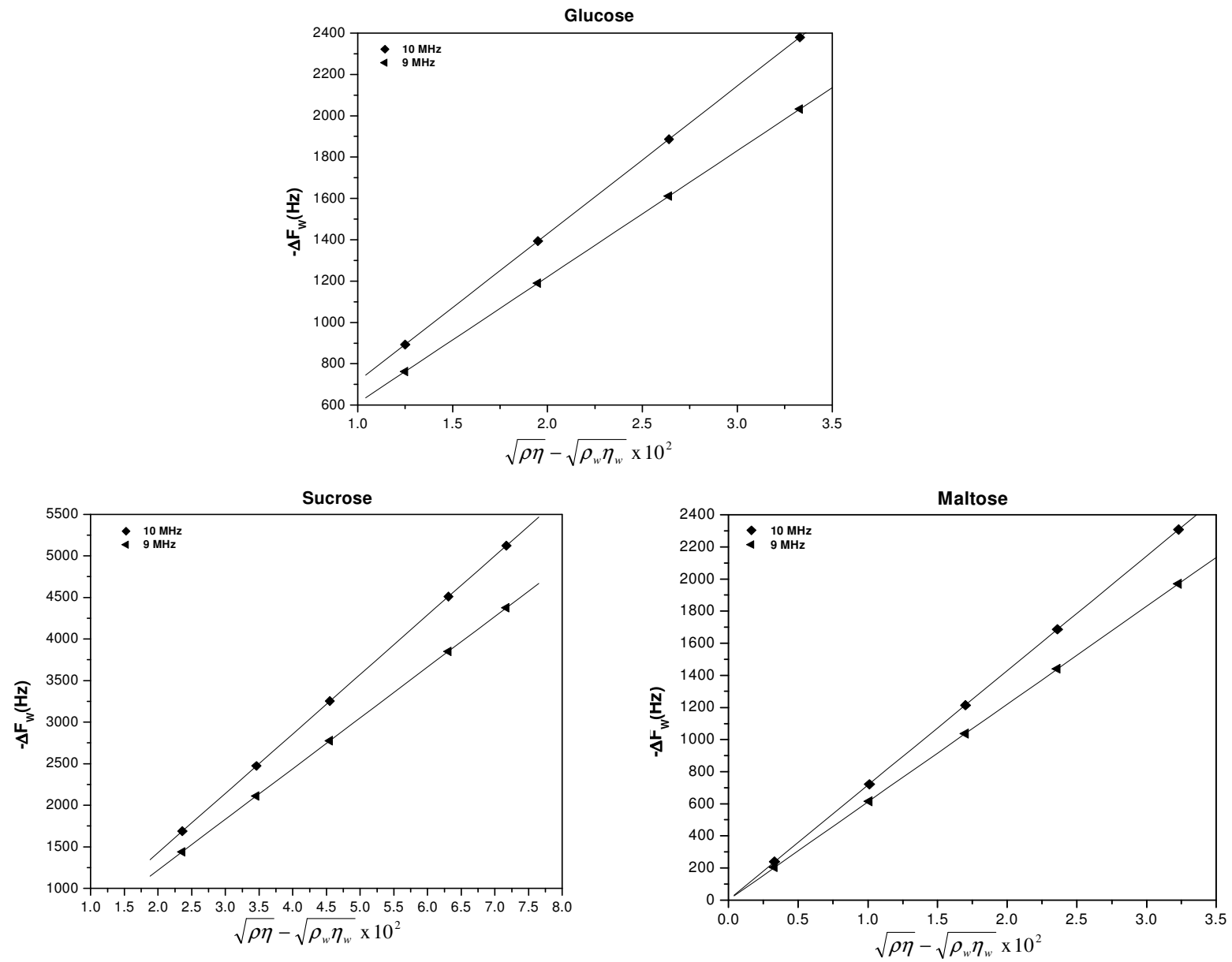

Fig. 3: The shift of $-\Delta f_{W}$ versus $\left(\sqrt{\rho \eta}-\sqrt{\rho_{W} \eta_{W}}\right) \times 10^{2}$ of sucrose, glucose and maltose for two kinds of crystal (9 $\mathrm{MHz}$ and $10 \mathrm{MHz}$ )

was measured and then acetone was applied to the crystal, followed by the application of a large volume of solvent in question.

To understand the relationship between the frequency change of the QCM immersed in solution and the properties of the liquid (density, $\rho$ and viscosity, $\eta$ ), results obtained by Kanazawa and Gordan $^{[3,4]}$ was referred. However, in this experiment, oscillation in distilled water was taken as a reference and a modified version the Kanazawa and Gordon equation was obtained and is given below ${ }^{[5]}$ :

$\Delta f_{W}=-2.26 \times 10^{-6} n f_{o}^{3 / 2}\left(\sqrt{\rho \eta}-\sqrt{\rho_{W} \eta_{W}}\right)$

where $\rho_{w}$ and $\eta_{w}$ represent the density and viscosity of water, respectively. Under the present condition,

$\sqrt{\rho_{W} \eta_{W}}=0.0944\left(\rho_{W}=0.997 \mathrm{gcm}^{-3}, \eta_{W}=0.894 \times 10^{-2} \mathrm{P}\right) \cdot$

Equation (1) were examined by changing $n, f_{b}, \rho$ and $\eta$.

\section{RESULT AND DISCUSSION}

Figure 2 shows the frequency shift of the quartz for gold-sucrose, gold-glucose and gold-maltose interfaces at concentration of 5-25 wt \% . We have observed that the experimental value of $-\Delta f_{W}$ for the aqueous saccharide increased linearly with the increasing of sample concentration. Similar trends were also observed by other scientist ${ }^{[2,5-7]}$ for sucrose and glucose solution. We also found that sucrose gave a higher frequency change compared to glucose and maltose for both 9 and $10 \mathrm{MHz}$ QCM. The increase in frequency shift is greatly due to the increment of the molecule adsorbed to the gold surface. The binding process changes the refractive index of the medium in contact with the metal surface. The different responses may due to the different formula structure of saccharide. Sucrose has the highest sensitivity because it has a longer diffusion path compare to other saccharide. In the other words, the more molecules are on the surface, the greater the frequency shift, $\Delta f_{W}$. If the concentration of the sample increases, the number of molecules 


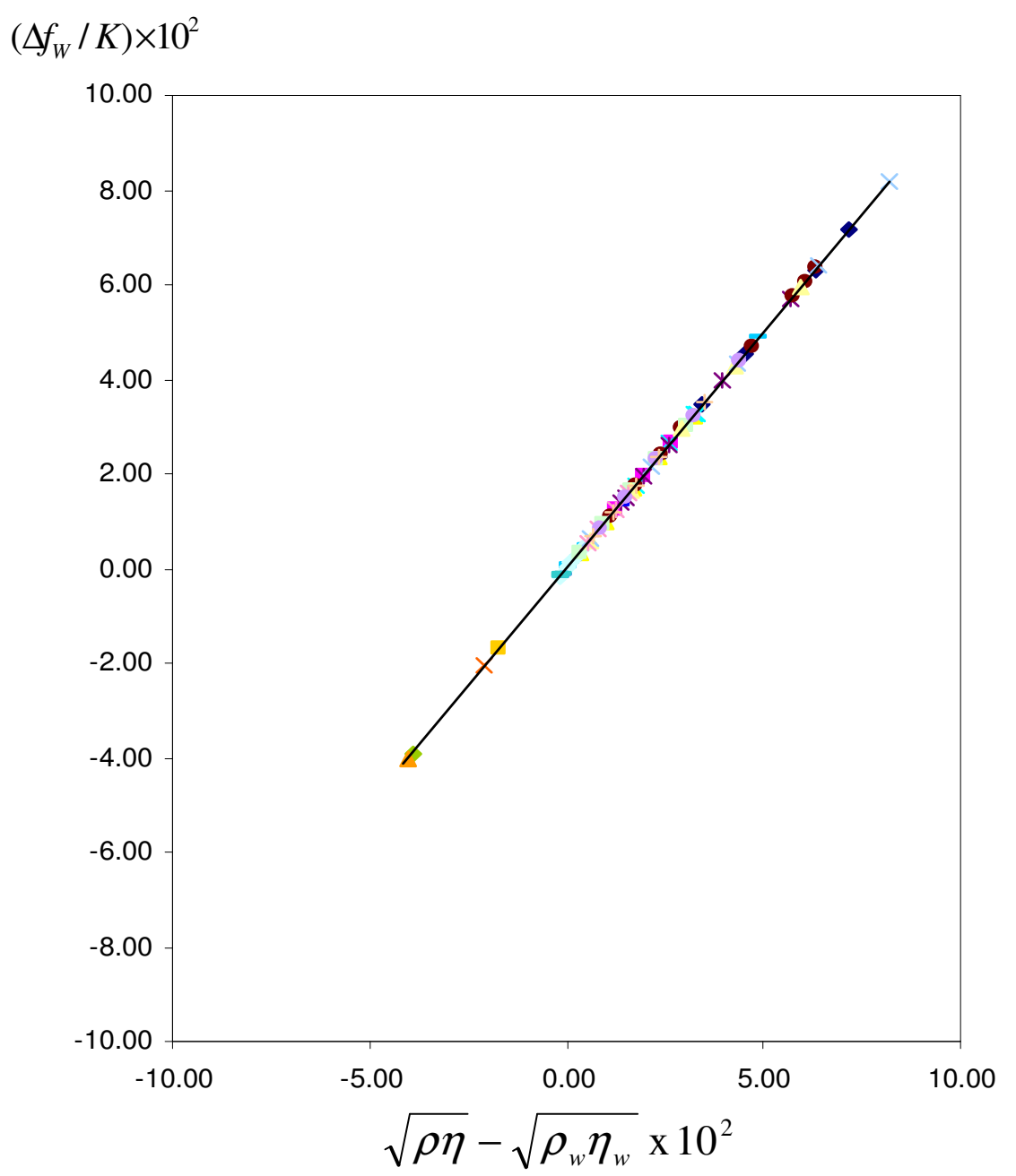

\begin{tabular}{|ll|}
\hline & Sucrose \\
& Glucose \\
$\times$ & Maltose \\
$*$ & $\mathrm{EtOH}$ \\
- & $1-\mathrm{PropOH}$ \\
+ & $\mathrm{NaCl}$ \\
- & $\mathrm{CaCl} 2$ \\
- & $\mathrm{AlCl} 3$ \\
& $\mathrm{PEG} 400$ \\
& PEG 4000 \\
& PEG 10000 \\
$\times$ & PEG 20000 \\
$*$ & Urea \\
- & Glycerol \\
+ & C2H6OS \\
- & G70 \\
- & G90 \\
& Acetone \\
$\square$ & Benzene \\
$\triangle$ & Hexane \\
$\times$ & Toluene \\
\hline & Linear (Sucrose) \\
\hline
\end{tabular}

Fig. 4: Proportionality between $\Delta f_{W} / K$ and $\sqrt{\rho \eta}-\sqrt{\rho_{W} \eta_{W}}$ for various solutions

increases and consequently the negative frequency shift become higher.

Various aqueous solutions were tested to verify Equation. (1). It was found that the oscillation frequency of the crystal depends on the physical parameter (density and viscosity) of the liquid properties. Figure 3 shows a plot of the frequency change against $\sqrt{\rho \eta}-\sqrt{\rho_{w} \eta_{w}}$ using aqueous sucrose, maltose and glucose for the $9 \mathrm{MHz}$ and $10 \mathrm{MHz}$ crystals where only one side of the crystal was expose to the solutions. The slope of the straight line that reflect the sensitivity of the detection of sucrose, glucose and maltose are $7.14 \times 10^{2}(10 \mathrm{MHz}), 6.10 \mathrm{x}$ $10^{2}(9 \mathrm{MHz}), 7.14 \times 10^{2}(10 \mathrm{MHz}), 6.10 \times 10^{2}(9 \mathrm{MHz})$ and $7.13 \times 10^{2}(10 \mathrm{MHz}), 6.09 \times 10^{2}(9 \mathrm{MHz})$ respectively. According to the theory, the plot of $\Delta f_{W} / K$ versus $\sqrt{\rho \eta}-\sqrt{\rho_{W} \eta_{W}}$ should give a line with a slope of unity. This is verified by the data on $\Delta f_{W} / K$ for aqueous sucrose, glucose and maltose plotted in Fig. 4.
A least square fit of the data gives a straight line between the points with slope of 1 for both $9 \mathrm{MHz}$ and $10 \mathrm{MHz}$ crystals. Aqueous saccharide were taken as a reference for all experimental samples because it gave the most effective detection sensitivity for quartz crystal microbalance in liquid.

For aqueous ethanol and methanol solutions, the value of $\sqrt{\rho \eta}$ does not necessarily increased with increased concentration and it is worth noting that the decrease in frequency corresponds well with $\sqrt{\rho \eta}$. The mass added or lost at the oscillator surface also does not experience any shear deformation during oscillation, but the exponentially damped shear wave is developed in solution at the surface affected the frequency of the QCM.

The response behavior of two different crystals to $\sqrt{\rho \eta}$ in different aqueous solutions was compared to investigate the factor influencing the frequency changes of the crystal. The relations between $f_{W}, \Delta f_{W} / K$ and 
Table 1: $\quad \Delta f_{W}$ in various aqueous solutions. $K$ values of the crystals were $6.10 \times 10^{4}(9 \mathrm{MHz})$ and $7.14 \times 10^{4} \mathrm{~cm}^{2} \mathrm{~g}^{-1} \mathrm{~s}^{-1 / 2}(10 \mathrm{MHz})$

\begin{tabular}{|c|c|c|c|c|c|c|c|}
\hline \multirow{2}{*}{\multicolumn{2}{|c|}{ Sample }} & \multirow{2}{*}{\multicolumn{2}{|c|}{ Concentration }} & \multirow{3}{*}{$\begin{array}{c}\left(\sqrt{\rho \eta}-\sqrt{\rho_{W} \eta_{W}}\right) \times 10^{2} \\
2.36\end{array}$} & \multirow{2}{*}{\multicolumn{2}{|c|}{$-\Delta f_{W}$}} & \multirow{3}{*}{$\begin{array}{c}-\Delta f_{w} / K \times 10^{2} \\
2.36\end{array}$} \\
\hline & & & & & & & \\
\hline \multirow{14}{*}{ 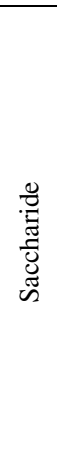 } & \multirow{5}{*}{$\begin{array}{c}\text { Sucrose } \\
\mathrm{C}_{12} \mathrm{H}_{22} \mathrm{O}_{11}\end{array}$} & 5 & \multirow{14}{*}{$\frac{2}{3}$} & & 1440.07 & 1686.48 & \\
\hline & & 10 & & 3.46 & 2111.29 & 2472.56 & 3.46 \\
\hline & & 15 & & 4.55 & 2776.41 & 3251.48 & 4.55 \\
\hline & & 20 & & 6.31 & 3850.36 & 4509.20 & 6.31 \\
\hline & & 25 & & 7.17 & 4375.13 & 5123.77 & 7.17 \\
\hline & \multirow{4}{*}{$\begin{array}{l}\text { Glucose } \\
\mathrm{C}_{6} \mathrm{H}_{12} \mathrm{O}_{6}\end{array}$} & 10 & & 1.25 & 762.75 & 893.27 & 1.25 \\
\hline & & 15 & & 1.95 & 1189.89 & 1393.49 & 1.95 \\
\hline & & 20 & & 2.64 & 1610.93 & 1886.58 & 2.64 \\
\hline & & 25 & & 3.33 & 2031.97 & 2379.66 & 3.33 \\
\hline & \multirow{5}{*}{$\begin{array}{c}\text { Maltose } \\
\mathrm{C}_{12} \mathrm{H}_{22} \mathrm{O}_{11}\end{array}$} & 5 & & 0.33 & 204.33 & 239.30 & 0.33 \\
\hline & & 10 & & 1.01 & 615.34 & 720.64 & 1.01 \\
\hline & & 15 & & 1.70 & 1036.77 & 1214.18 & 1.70 \\
\hline & & 20 & & 2.36 & 1440.83 & 1687.37 & 2.36 \\
\hline & & 25 & & 3.23 & 1971.05 & 2308.31 & 3.23 \\
\hline \multirow{12}{*}{$\underset{\frac{0}{2}}{\stackrel{0}{0}}$} & \multirow{4}{*}{$\begin{array}{c}\text { Sodium Chloride, } \\
\mathrm{NaCl}\end{array}$} & 0.1 & \multirow{12}{*}{$\sum_{\Xi}$} & $\begin{array}{l}-0.08 \\
\end{array}$ & -46.49 & -52.37 & -0.08 \\
\hline & & 1 & & 0.03 & 17.51 & -9.60 & 0.03 \\
\hline & & 10 & & 0.10 & 63.74 & 43.78 & 0.10 \\
\hline & & 100 & & 0.54 & 328.16 & 350.94 & 0.54 \\
\hline & \multirow{4}{*}{$\begin{array}{c}\text { Calcium Chloride, } \\
\mathrm{CaCl}_{2}\end{array}$} & 0.1 & & -0.11 & -65.63 & $\begin{array}{l}-155.19 \\
\end{array}$ & -0.11 \\
\hline & & 1 & & 0.03 & 17.39 & -11.24 & 0.03 \\
\hline & & 10 & & 0.18 & 111.92 & 192.98 & 0.18 \\
\hline & & 100 & & 1.35 & 824.86 & 904.23 & 1.35 \\
\hline & \multirow{4}{*}{$\begin{array}{c}\text { Aluminium } \\
\text { Chloride, } \\
\mathrm{AlCl}_{3}\end{array}$} & 0.1 & & 0.05 & 27.58 & -176.04 & 0.05 \\
\hline & & 1 & & 0.06 & 35.85 & -89.11 & 0.06 \\
\hline & & 10 & & 0.47 & 286.72 & 219.00 & 0.47 \\
\hline & & 100 & & 4.89 & 2980.94 & 3539.39 & 4.89 \\
\hline \multirow{20}{*}{ 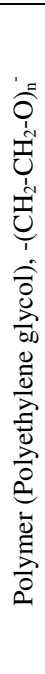 } & \multirow{5}{*}{ PEG 400} & 0.5 & \multirow{20}{*}{$\bar{\nabla}$} & -0.18 & -107.25 & -125.60 & -0.18 \\
\hline & & 1.5 & & 0.04 & 27.40 & 32.09 & 0.04 \\
\hline & & 2.5 & & 0.11 & 65.86 & 77.13 & 0.11 \\
\hline & & 3.5 & & 0.22 & 136.53 & 159.89 & 0.22 \\
\hline & & 4.5 & & 0.42 & 255.29 & 298.97 & 0.42 \\
\hline & \multirow{5}{*}{ PEG 4000} & 0.5 & & 0.33 & 202.12 & 236.71 & 0.33 \\
\hline & & 1.5 & & 0.92 & 563.66 & 660.11 & 0.92 \\
\hline & & 2.5 & & 1.64 & 1001.78 & 1173.20 & 1.64 \\
\hline & & 3.5 & & 2.31 & 1407.36 & 1648.17 & 2.31 \\
\hline & & 4.5 & & 3.03 & 1847.93 & 2164.13 & 3.03 \\
\hline & \multirow{5}{*}{ PEG 10000} & 0.5 & & 0.60 & 364.33 & 426.67 & 0.60 \\
\hline & & 1.5 & & 1.67 & 1017.55 & 1191.67 & 1.67 \\
\hline & & 2.5 & & 2.95 & 1798.80 & 2106.59 & 2.95 \\
\hline & & 3.5 & & 4.28 & 2610.14 & 3056.76 & 4.28 \\
\hline & & 4.5 & & 5.93 & 3620.42 & 4239.92 & 5.93 \\
\hline & \multirow{5}{*}{ PEG 20000} & 0.5 & & 0.63 & 382.56 & 448.02 & 0.63 \\
\hline & & 1.5 & & 2.13 & 1299.99 & 1522.44 & 2.13 \\
\hline & & 2.5 & & 4.31 & 2632.61 & 3083.08 & 4.31 \\
\hline & & 3.5 & & 6.38 & 3895.10 & 4561.59 & 6.38 \\
\hline & & 4.5 & & 8.19 & 4999.64 & 5855.13 & 8.19 \\
\hline
\end{tabular}

$\sqrt{\rho \eta}-\sqrt{ } \rho_{\mathrm{w}} \eta_{\mathrm{w}}$ with these crystals in various solution are illustrated in Table 1 . It was found that $-\Delta f_{w}$ increased with increasing $f_{o}$ (fundamental frequency). The 10 $\mathrm{MHz}$ crystals are more sensitive to the mass changes but were found to be less stable compared to $9 \mathrm{MHz}$ crystal.

\section{CONCLUSION}

We have carried out our QCM measurements with some aqueous solutions. An uncoated quartz crystal was used for monitoring changes of viscosity and density of the liquid simultaneously. Our results implies that the frequency change is proportional to $\sqrt{ } \rho \eta-\sqrt{ } \rho_{\mathrm{w}} \eta_{\mathrm{w}}$. With the QCM principle used in liquids, it is possible to detect properties of liquids up to the ppm range. From the experimental results, we also can determine a solute in a selected solution. The relations between the frequency shift, $\Delta f_{w}$ and $V_{\rho \eta}$ are reported. The above results are very helpful for understanding the frequency behavior in contact with liquid phase and offer new potential routes for studying liquid properties in the future. 


\section{ACKNOWLEDGMENT}

The authors would like to acknowledge the financial support provided by the Malaysian Government through the IRPA (Vote Number: 09-0204-0458-EA001) program.

\section{REFERENCES}

1. Bruckenstein, S. and M. Shay, 1985. Experimental aspects of use of the quartz crystal microbalance in solution. Electrochimica Acta, 30: 1295-1300.

2. Kanazawa, K.K. and J.G. Gordon II, 1985. Frequency of a quartz microbalance in contact with liquid. Anal. Chem., 57: 1770-1771.
3. Konash, P.L. and G.J. Bastiaans, 1980. Piezoelectric crystals as detectors in liquid chromatography. Anal. Chem., 52: 1929.

4. Kanazawa, K.K. and J.G. Gordon II, 1985. The oscillation frequency of a ouartz resonator in contact with a liquid. Analytical Chimica Acta, 175: 99-105.

5. Kurosawa, S., E. Tawara, N. Kamo and Y. Kobatake, 1990. Oscillating frequency of piezoelectric quartz crystal in solutions. Analytica Chimica Acta, 230: 41-49.

6. Shana, Z.A., D.E. Radtke, U.R. Kelkar and F. Josse, 1990. Theory and application of quartz resonator as a sensor for viscous liquids. Analytica Chimica Acta, 231: 317-320.

7. Nomura, T. and T. Yanagihara, 1991. Electrodeseparated piezoelectric quartz crystal and its application as a detector for liquid chromatography. Analytica Chimica Acta, 248: 329-335. 